\title{
The Acceptance, Concerns, and Obstacles related to COVID-19 Vaccination among the General Society in Saudi Arabia
}

\author{
Murouj A. Almaghrabi ${ }^{1}$, Wejdan E. Rahali ${ }^{1}$, Ahad S. Babkier ${ }^{1}$, Sadeem E. Bukhari ${ }^{1}$, Mokhtar M. Shatla ${ }^{2}$. \\ ${ }^{1}$ Department of Medicine, College of Medicine, Umm Al-Qura University, Makkah, Saudi Arabia. \\ ${ }^{2}$ Associate professor, Department of Community Medicine and pilgrimage health, Faculty of Medicine, Umm Al-Qura University, Makkah, Saudi
} Arabia.

\section{ABSTRACT}

Background: The strongest COVID-19 control solution is an effective vaccine. Several factors affect COVID-19 vaccine acceptance by the general population. Public health officials and practitioners must recognize all obstacles that may discourage people from obtaining COVID-19 vaccines. The present study aimed to measure the extent of community acceptance of taking the COVID-19 vaccine in Saudi Arabia, as well as to assess the limitations, concerns, and obstacles that is related to the vaccine.

Methods: A cross-sectional study of a sample consisting of 1400 participants living in Saudi Arabia assessed socio-demographic characteristics, general characteristics of the vaccinated participants, and participants' acceptance, concerns, and obstacles related to COVID-19 vaccination.

Results: Most participants were females (63.1\%) and from the western region of Saudi Arabia (81.2\%). Nearly equal number was reported between vaccinated and non-vaccinated participants with COVID-19 vaccination (45\% and 55\% respectively). Exact of 324 participants reported obstacles that prevent them from getting the vaccine, in which the highly reported item was lack of information about the vaccine (21.6\%). A total number of 1,796 participants reported few concerns, in which the most common item was health concern (43.4\%), followed by misleading ideas and beliefs (35.4\%). A recorded statistical significance p-value was determined between the participants who received COVID-19 vaccination and the gender, nationality, place of residence, and working in government sector ( $\mathrm{P}=0.002,0.002,0.001$, and 0.011 , respectively).

Conclusions: Awareness of COVID-19 vaccination is still lacking. Thus, a targeted health educational programs are needed to increase the individuals convince and reduce the fears about the vaccination.

Keyword: Acceptance, COVID-19, obstacles, Saudi Arabia, vaccination.

\section{Introduction}

Coronavirus disease 2019 (COVID-19) is a highly contagious respiratory illness caused by a novel Severe Acute Respiratory Syndrome Coronavirus 2 (SARS-CoV-2) coronavirus. The World Health Organization (WHO) officially declared COVID-19 to be a pandemic on March 11, 2020. COVID-19 was first reported in Wuhan, China [1] and has since rapidly spread globally in a short period of time [2] because the virus is extremely infectious, it spreads quickly and evolves over time in humans [1].

\begin{tabular}{|l|l|}
\hline \multicolumn{2}{|c|}{ Access this article online } \\
\hline Quick Response Code: & Website: \\
& www.smh-j.com \\
\cline { 2 - 2 } & DOI: \\
\hline
\end{tabular}

Virus transmission can happen via human-to-human contact or through droplet transfer, cough, or sneeze $[3,4]$. The goal of preventative measures is to limit and control the spread of the infection. Social distancing, such as stopping large public or private meetings [2], widespread closures of public and commercial spaces [5], wearing masks and gloves, and avoiding unnecessary travels and visits, are all ways to decrease the risk of infection [2]. While efforts to promote social distancing and masking have been effective,

Address for correspondence: Murouj A. Almaghrabi, Department Of Medicine, College of Medicine, Umm Al-Qura University, KSA. E-mail: murouj@ outlook.com

Received: 1 November 2021 | Accepted: 22 November 2021

This is an open access article by SMHJ is licensed under Creative Commons Attribution 4.0 International License.

(https://creativecommons.org/licenses/by/4.0)

Please cite this article as: Rahali W, Almaghrabi M, Babkier A, Bukhari S, Shatla M. The Acceptance, Concerns, and Obstacles related to COVID-19 Vaccination among the General Society in Saudi Arabia: COVID-19 vaccination perception among the public. SMHJ [Internet]. 2021;1(1):14-25.

(C) Saudi Medical Horizons Journal

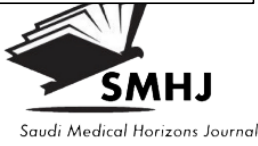


They have had economic and psychological consequences. The strongest COVID-19 control solution is an effective vaccine [5], therefore vaccination may be used to prevent infection or to lessen the severity of the disease, as well as viral shedding and transmission [6], there are 30 vaccines in clinical trials as of late August 2020, with over 200 in different stages of development [7]. In Saudi Arabia, there are two different types of vaccination used. Firstly, the non-replicating viral vectors (NRVV) from University of Oxford/AstraZenec Organization, Adenovirus-based vaccines are the most commonly used virally vectored candidates for non-replicating SARS-CoV-2 vaccines, after injection NRVV vaccines caused minor local responses, such as swelling and redness, as well as systemic symptoms such as malaise, fatigue, fever, and headache, which all went away within 96 hours. The second type is messenger ribonucleic acid (mRNA) vaccine from BioNTech/Fosun Pharma/Pfizer Organization. Nucleic acid vaccine candidates are usually quicker and less expensive to produce than protein subunit vaccines, so many mRNA-based SARS-CoV-2 vaccine candidates have been created, although these vaccines caused mild side effects after the first dose, such as fatigue, chills, headache, myalgia, and localized pain at the injection site, side effects worsened as the dosage was increased, and booster doses in some patients caused moderate to serious local and systemic responses [8]. Hence, public health practice suggests that a sufficient number of people must be vaccinated to control an outbreak like COVID-19 [5]. A previous study has surveyed 13,426 people in 19 countries in June 2020 to see what factors affect COVID-19 vaccine acceptance. In which 71.5 percent of respondents showed they would be very or very likely to get the COVID-19 vaccine [9]. Public health officials and practitioners must recognize all obstacles that may discourage people from obtaining the approved vaccines. Infectious disease control in the United States (US) and around the world has established a wide range of obstacles that can obstruct vaccine uptake, resulting in disease control failure. The barriers were separated into two types: structural and psychological. First: structural barriers, which are the structural problems that can make it difficult for individual people to get vaccines: Time, transportation, expense, and clinic or outlet location are examples of structural barriers that affect one's ability to access a service. Second: the attitudinal barriers, which are the attitudes or expectations that affect at-risk individuals' ability to seek out and/or accept services. They have also included the beliefs about the communicable disease, vaccine beliefs, fear, and trust in healthcare and government agencies in the sense of vaccination [5]. Early detection of the population acceptance towards COVID-19 vaccination is necessary, which directly affect the quality of life for the community. To the best of our knowledge, there is no study in Saudi Arabia that has previously discussed this issue. Since there is a remarkable number of people did not receive the vaccine of COVID-19 in the Kingdome of Saudi Arabia (KSA) population although reservation is available for vaccination for everyone. Therefore, assessing this situation in depth is required. The present study aims to measure the extent of community demand for taking the COVID-19 vaccine in KSA, as well as to assess the limitations, concerns, and obstacles that is related to it.

\section{Methods}

We conducted this online-based cross-sectional study for citizens and residents in Saudi Arabia. A sample of 1400 individuals was collected randomly through a self-administered online questionnaire from 18 March 2021 to 24 March 2021. All individuals who are eligible to answer the questionnaire were invited to participate in the study. We have excluded individuals below the age of 15 years. We used OpenEpi version 3.0 [10] to calculate the required sample size for this study. In consideration of the following: the population size of Saudi Arabia residents is about $35,013,414$ people [11].Keeping the Confidence Interval (CI) level at $99 \%$ and considering anticipated $\%$ of frequency as $50 \%$ and taking design effect as 1.0. The sample size was 664 participants. In case of data loss, a total sample size of 700 participants was required. However, the final collected data included 1,400 participants. The questionnaire of the present study has been developed by reviewing the previous literature. Then, it was sent to a group of certified doctors in the speciality to check its validity. This questionnaire consists of several items distributed over three main parts: 1) First: general characteristics of the participants, including gender, age, nationality, educational level, occupation, residence place, past medical history including previous history of COVID19 infection, and the received vaccines. 2) Second: four items to discover the general characteristics of the vaccinated participants. 3) Last part: five items to assess the participants' acceptance, concerns, and obstacles related to COVID-19 vaccination.

Ethical approval of this study was obtained from the Institutional Review Board (IRB) at Umm Al-Qura 
University (UQU), Makkah, Saudi Arabia (code HAPO-02-K-012-2021-08-722). Prior to answering the questionnaire, electronic informed consent was gained from each participant, and they were informed that it is a voluntary effort, and they can withdraw at any time. The confidentiality of the participants was ensured. No personal data (such as names or phone numbers) were obtained from any participant.

\section{Statistical analysis}

The data analysis was performed with Statistical Package for the Social Sciences (SPSS), version 22 (IBM, Armonk, NY, USA). The study graphs were performed using Microsoft Excel software. All statistical analyses were performed using two-tailed tests with an alpha error of 0.05. Statistical significance was set at $\mathrm{P}<0.05$. We used frequencies and percentages to describe all categorical data of the participants. Crosstabulation was performed between reviving COVID-19 vaccination and demographic data of the participants to detect the most significant variables based on Pearson's chi-squared test.

\section{Results}

Socio-demographic characteristics of the participants A total number of 1400 participants were included in our study. (Table 1) demonstrates the sociodemographic characteristics of the participants in which nearly half of them were between the ages of 16-36 years $(680,48.6 \%)$, with females predominate $(884,63.1 \%)$. The majority of responders were Saudis $(1314,93.9 \%)$ while only view numbers were recorded from non-Saudi participants $(86,6.1 \%)$. Most of the participants were from the western region $(1137,81.2 \%)$, not known to have any illness $(1,034$, $73.2 \%)$, and with an educational level of bachelor's degree $(781,55.8 \%)$. Regarding occupation, exact number of 486 participants were Retired/ non-worker/ Housewife $(34.7 \%)$, followed by 347 students $(24.8 \%)$. The least recorded occupation in our population was a private job $(32,2.3 \%)$. Close percentages were observed between workers in the government sector and those not working in it $30.6 \%$ and $28.6 \%$, respectively). Among all respondents, only view numbers have recorded a previous history of COVID-19 infection (204, 14.6\%) while the majority have no history of infection $(1196,85.4 \%)$. (Figure 1) represents the participants' commitment regarding different types of vaccinations. In which more than half of the participants were not restricted in receiving flu vaccine annually $(965,69 \%)$ in comparison to 435 participants who were restricted to receive the flu vaccine annually $(31 \%)$. In addition, most of the participants does not receive the meningitis vaccine every 5 years $(1,233,88 \%)$ while only a view number of participants were committed to receive it $(167,12 \%)$. In contrary to COVID-19 vaccination, close numbers were recorded between vaccinated and non-vaccinated participants. Hence, non-vaccinated participants were slightly higher in number compared to vaccinated participants ( 775 , $55 \%$ and $625,45 \%$, respectively). General characteristics of the vaccines and their side effects Our study recorded a total of 625 vaccinated participants. (Table 2) presents the general characteristics of the vaccines and their side effects among the participants. In which, number of vaccinated participants with Pfizer were 325 (52\%), while AstraZeneca-Oxford were 300 participants (48\%). The Purpose of getting vaccinated varied between participants, the most reason was desire and conviction $(548,87.7 \%)$ followed by vaccination affordability $(12,1.9 \%)$, some for their job requirement $(30,4.8 \%)$ others for their family desire $(9,1.4 \%)$ also as a traveling requirement $(22,3.5 \%)$, then as a protection for the family $(1,0.2 \%)$, additionally for preventing covid-19 complication (1, $0.2 \%)$, and for multiple purposes $(1,0.2 \%)$, and lastly for no purpose $(1,0.2 \%)$. According to the vaccine side effects, more than a half of the participants suffered from the presence of side effects (343, $54.9 \%$ ), and the others did not have any side effects $(282,45.1 \%)$. Side effects have been categorized into generalized and systematic, considering generalized it was fever $(232,67.7 \%)$, body tremor $(76,22.2 \%)$, fainting $(3,0.9 \%)$, uterine pain $(1,0.3 \%)$, swelling of mouth and face and neck $(6,1.7 \%)$. Systematically side effects were recognized as follows, dermatological (231, 67.3\%), gastrointestinal (129, $37.6 \%)$, neurological $(249,72.6 \%)$, respiratory $(21$, $6.2 \%)$, and musculoskeletal $(282,82.3 \%)$.

\section{Acceptance, concerns, and obstacles related to the vaccination}

Exact number of 371 participants were accepted the idea of coronavirus vaccination $(47.9 \%)$. On the other hand, 142 participants were refusing it (18.3\%). In regard to the barriers, $599(77.3 \%)$ participants have no barriers to taking the vaccine and 176 (22.7\%) having barriers. A $262(33.8 \%)$ are still confused about taking the vaccination. (Table 3) Summarize the results. (Table 4) represents the number of participants who reported obstacles that prevented them of getting the vaccine. From a total of 324 response, the most report obstacle was lack of information and education about COVID-19 and its vaccination and about how to use the application $(70,21.6 \%)$, followed by 
The Acceptance, Concerns, and Obstacles related to COVID-19 Vaccination among the General Society in Saudi Arabia.

conflicting information concerning the vaccine's safety $(62,19.1 \%)$, then followed by having health problems or allergy $(58,17.9 \%)$, after that multiple available types of vaccination (46, $14.2 \%)$, then taking a long time to obtain an appointment and being far away from the centres $(29,9.0 \%)$. The least reported obstacles were 'others' $(7,2.2 \%)$, followed by Covid19 was previously diagnosed in less than 6 months $(8$, $2.5 \%)$, then Parental refuse $(12,3.7 \%)$, after that do not have a health reason to be vaccinated $(15,4.6 \%)$, followed by lactating and pregnant women $(17,5.2$ $\%$ ). The total number of concerns reported by the participants were 1,796 , most common concern reported by them was a health concern $(779,43.4 \%)$, followed by misleading ideas and beliefs $(635,35.4 \%)$, followed by vaccine trial and efficacy $(213,11.8 \%)$ and the least common concerns reported by participants was information circulated on social media $(106,5.9 \%)$, followed by virus strains $(63,3.5 \%)$. All results are shown in (Table 5).

\section{Association between receiving COVID-19 vaccination and demographic data}

(Table 4) demonstrates the association between receiving COVID-19 vaccination and the demographic characteristics of the participants. Among participants who did not receive the vaccine, females recorded higher numbers compared to male participants (67.4\% and $32.6 \%$, respectively). On the other hand, females were detected to receive the vaccine more than males $(57.9 \%$ and $42.1 \%$, respectively) with a recorded statistical significance $(\mathrm{P}=0.002)$. As for nationality, $96.5 \%$ of the vaccinated participants were Saudis compared to $3.5 \%$ of nonSaudis, with statistical significance $(\mathrm{P}=0.002)$. Regarding place of residence, $85.1 \%$ of the vaccinated participants were from western region followed by $11.5 \%$ of the vaccinated participants from central region. On the other hand, $78.1 \%$ of the western region were non vaccinated with historical statistical significance $(\mathrm{P}=0.001)$. Other demographic variables associated with receiving COVID-19 vaccination are shown in (Table 6).

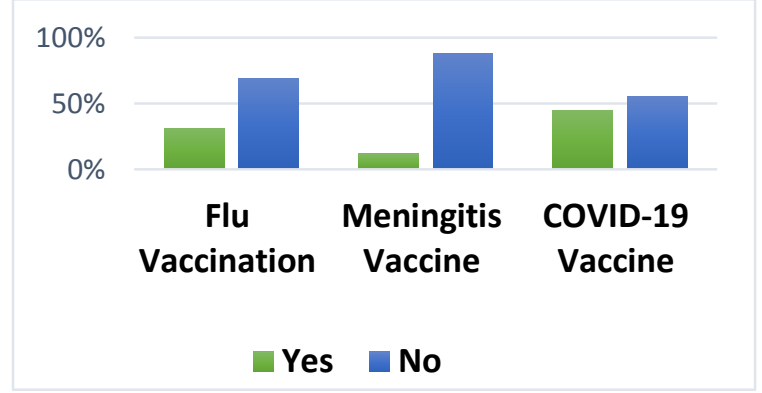

Figure 1: Participants Practice Regarding Different Vaccinations $(n=1400)$.

\section{Discussion}

The current study is among the first studies that represented an assessments of COVID-19 vaccination behavior in KSA. In which the study revealed almost the same number between vaccinated and nonvaccinated participants. The most common obstacle of COVID-19 vaccination reported by non-vaccinated participants is their belief of conflicting information concerning the vaccine's safety. While the health concerns reported to be the most concerns that prevent people to take the vaccine.

\section{Socio-demographics of the participants}

The participants' distribution on the two genders was semi-equal with predominance to the female gender. As most of our participants were reported to be Saudis (93.9\%), generalizability of the current results will be more accurate on the Saudis sample not residents of different nationalities in the KSA. Participants were included from all regions of KSA, and various ages ranged from 16 years to more than 57 years were also included in the study which will contribute to extracting different opinions from different generations. However, the vast majority of the study's participants were highly educated which may affect generalizing the results. We recommend further studies to be conduct on low educated populations in Saudi Arabia to better understand their opinion, acceptance, concerns, and obstacles related to COVID-19 vaccination.

General characteristics of the vaccinated participants and the reported side effects

We gathered data from people who got either OxfordAstraZeneca or Pfizer BioNTech COVID-19 vaccinations in Saudi Arabia, total of 625 received AstraZeneca-Oxford 325 (52\%) and Pfizer 300 (48\%) vaccines. In our survey, $54.9 \%$ said they had suffered some side effects and $282(45.1 \%)$ they didn't have any side effects. The most prevalent adverse effects were fatigue (72.9\%), fever (67.6\%), pain in site of injection $(61.5 \%)$, and headache $(55.1 \%)$, after that nausea (29.7\%). Unlike other studies, in UK they discovered that systemic side effects, such as headache and fatigue, impacted fewer than one out of every four people and were less prevalent in the population than clinical studies predicted [12]. In our study, most of the participants who took the Oxford-AstraZeneca vaccine reported considerably more side effects than those who received the Pfizer-BioNTech vaccine, according to our findings. As can also be seen in recent study done in Saudi Arabia [13].

\section{Acceptance of COVID-19 vaccine}


As reported previously in the current study, six hundred twenty-five individuals $(44.6 \%)$ have been vaccinated while a total of 775 individuals $(55.4 \%)$ have not been vaccinated and they were divided into $371(26.5 \%)$ who were willing to take the vaccine, 262 (18.7\%) might take the vaccine, and $142(10.2 \%)$ are refusing the vaccine (Table 7).

In comparison to our study, a previous study in the US reported that $450(67 \%)$ of 672 respondents would accept the COVID-19 vaccine. Despite the fact that a COVID-19 vaccine was accepted by $67 \%$ in the trial, there were significant demographic and geographic differences in vaccination acceptance [14]. Further study in Saudi Arabia included 992 attendees from four major cities (Riyadh, Jeddah, Dammam, and Abha) as well as some minor cities. Of the 992 attendees, $642(64.7 \%)$ accepted the COVID-19 vaccine, $70(7.0 \%)$ rejected the vaccine, and 280 $(28.2 \%)$ were hesitant about COVID-19 vaccine if it is available [15].

\section{Barriers of receiving COVID-19 vaccine}

From a total of $599(77.3 \%)$ individuals who were non-vaccinated, exact of $775(55.4 \%)$ had no barriers for taking the vaccine while $176(22.7 \%)$ had some. Vaccine variety types and COVID-19 strains issues 62 $(35.2 \%)$ was the major barrier effecting the participants, vaccine production issues $1(0.6 \%)$, Medication Issues $1(0.6 \%)$ and Religious Believes 1 $(0.6 \%)$ were the least barriers affecting them see (Table 8) for more details.

Previous evidence placed in Tanta and Kafrelsheikh Universities in Egypt among medical students, The most relevant barriers of COVID-19 vaccination were insufficient information regarding adverse effects of the vaccine (potential $74.2 \%$ and unknown $56.3 \%$ ), insufficient information regarding the vaccine itself (72.8\%), financial cost hindrance if the vaccine is not free $(68.0 \%)$, and insufficient trust in the vaccination source $(55.1 \%)$ [16].

\section{Concerns of COVID-19 vaccine}

The most popular concern in this study respectively, Harmful and non-beneficial issue 388 (77.6\%) Severe side effects $282(52.5 \%)$ Trials aren't over $208(38.7 \%)$ Leads to other health conditions $192(35.7 \%)$ Leads to death 135 (25.1\%) Effects Genes 116 (21.6\%) Only a trial $113(21 \%)$ Fear from Social Media trending 106 (19.7\%) Aimed to eliminate humans 78 (14.5\%) New strains of COVID-19 61 (11.3\%) Profit vaccine 57 (10.6\%) Becomes an annual vaccine 49 (9.1\%) Allergy or other health condition $5(1 \%)$ Strange disease and vaccine existence $3(0.6 \%)$ No suitable vaccine yet $1(0.2 \%)$ Fear of being infected while vaccinated $1(0.2 \%)$.

\section{Association between receiving COVID-19 vaccination and demographic data}

As shown in Table 6, a statistically significant association were reported between the gender and the demand for receiving COVID-19 vaccination as the females were predominated group $(884,63.1 \%)$. The reason may be due to the female keenness to take precautions and adhere to preventive measures and their sense of responsibility, as many studies reviled the same results even with different health issues [17-

19]. Understanding the sociodemographic characteristics in relation to COVID-19 vaccination receiving may aid in increasing global immunization program uptake to combat future pandemics. Hence, the socio-demographic characteristics of the current study were predominated group between the ages of $16-36$ years $(680,48.6 \%)$ followed by the ages of $37-$ 56 years $(535,38.2 \%)$ followed by 57 years and more $(185,13.2 \%)$. The participants were from all throughout Saudi Arabia., most of them were from the western region $(1,137,81.2 \%)$, followed by Central Region $(159,11.4 \%)$ and Eastern Region $(45,3.2 \%)$ and Southern Region $(35,2.5 \%)$ and Northern Region $(24,1.7 \%)$. As reported previously, the educational level of the participants was mostly bachelor's degree (781, 55.8\%) followed by High school/ diploma $(377,26.9 \%)$ and master or higher degree $(192,13.7 \%)$. Only minor percentage of participants were elementary/ intermediate School (50,3.6\%). As a comparison, the recent study that has been conducted in the four major cites (Riyadh - Dammam - Jeddah Abha) in Saudi Arabia [15] reported the sociodemographic characteristics of a number of 992 participants, they reported the predominate group to be between the ages of 26-35 years $(436,43.9 \%)$ followed by ages of $18-25$ years $(264,26.6 \%)$, ages of $36-45$ years $(230,24 \%)$ and ages 45 and more $(53,5.3 \%)$. Most of their responses were graduate individuals $(497,50.1 \%)$, post-graduate $(199,20 \%)$, followed by diploma $(153,15.4 \%)$, and high school students $(143,14.4 \%)$. The variety of those populations revealed almost the same conclusion which states that educational health programs are needed to increase the awareness of the safety of COVID-19 vaccination among the community individuals. In other recent study showed the different in acceptance rate between countries [20], it was previously reported in China (91.3- 72.6\%), Indonesia (93.3\%), Malaysia (94.3\%) Denmark (80.0\%), UK (90.1-64.0\%), Canada (68.7$80.0 \%)$, Turkey $(69.0 \%)$ Nigeria $(65.2 \%)$. 
The Acceptance, Concerns, and Obstacles related to COVID-19 Vaccination among the General Society in Saudi Arabia.

Table 1: Socio-demographics and sample

\begin{tabular}{|c|c|c|c|}
\hline Socio-demographic data & & No & $\%$ \\
\hline \multirow{2}{*}{ Gender } & Female & 884 & $63.1 \%$ \\
\hline & Male & 516 & $36.9 \%$ \\
\hline \multirow{3}{*}{ Age } & 16-36 years & 680 & $48.6 \%$ \\
\hline & 37-56 years & 535 & $38.2 \%$ \\
\hline & $57+$ years & 185 & $13.2 \%$ \\
\hline \multirow{2}{*}{ Nationality } & Saudi & 1,314 & $93.9 \%$ \\
\hline & Non-Saudi & 86 & $6.1 \%$ \\
\hline \multirow{4}{*}{ Past Medical History (PMH) } & None & 1,034 & $73.2 \%$ \\
\hline & Endocrine Conditions $*$ & 253 & $17.9 \%$ \\
\hline & Respiratory Conditions $* *$ & 72 & $5.1 \%$ \\
\hline & Others $* * *$ & 53 & $3.8 \%$ \\
\hline \multirow{5}{*}{ Place of Residence } & Western Region & 1,137 & $81.2 \%$ \\
\hline & Central Region & 159 & $11.4 \%$ \\
\hline & Eastern Region & 45 & $3.2 \%$ \\
\hline & Southern Region & 35 & $2.5 \%$ \\
\hline & Northern Region & 24 & $1.7 \%$ \\
\hline \multirow{4}{*}{ Educational level } & Elementary/ Intermediate School & 50 & $3.6 \%$ \\
\hline & High school/ Diploma & 377 & $26.9 \%$ \\
\hline & Bachelor's degree & 781 & $55.8 \%$ \\
\hline & Master or higher degree & 192 & $13.7 \%$ \\
\hline \multirow{6}{*}{ Occupation } & Student & 347 & $24.8 \%$ \\
\hline & Retired/non-worker/ Housewife & 486 & $34.7 \%$ \\
\hline & Health care provider & 118 & $8.4 \%$ \\
\hline & Office work & 313 & $22.4 \%$ \\
\hline & Field work & 104 & $7.4 \%$ \\
\hline & Private job & 32 & $2.3 \%$ \\
\hline \multirow{3}{*}{ Work in a Government Sector } & Yes & 429 & $30.6 \%$ \\
\hline & No & 400 & $28.6 \%$ \\
\hline & Do not work & 571 & $40.8 \%$ \\
\hline \multirow{2}{*}{ Previous history of COVID-19 } & Yes & 204 & $14.6 \%$ \\
\hline & No & 1,196 & $85.4 \%$ \\
\hline
\end{tabular}

* Endocrine conditions: including Hypertension (HTN), Diabetes Mellitus (DM), Thyroid

Diseases, and Obesity.

** Respiratory Conditions: Asthma and Allergic rhinitis.

*** Others: including cancers, rheumatoid, Food Allergy, Osteoporosis, Brucellosis, Peptic

ulcer, Epilepsy, Multiple Sclerosis, Parkinson, Heart Failure, Chronic Kidney Failure, Cirrhosis,

Psychiatric condition, Psoriasis, and Sickle cell anemia.

characteristics of the participants $(n=1400)$. 
The Acceptance, Concerns, and Obstacles related to COVID-19 Vaccination among the General Society in Saudi Arabia.

Table 2: General characteristics of the vaccines and their side effects $(n=625)$.

\begin{tabular}{|c|c|c|c|}
\hline \multicolumn{2}{|c|}{ General characteristics and side effects } & \multirow{2}{*}{$\frac{\text { No. }}{325}$} & \multirow{2}{*}{$\begin{array}{c}\% \\
52 \%\end{array}$} \\
\hline Trung of Vosing & Pfizer & & \\
\hline Iype or V accine & AstraZeneca-Oxford & 300 & $48 \%$ \\
\hline \multirow{9}{*}{ Purpose of getting vaccinated } & Desire and conviction & 548 & $87.7 \%$ \\
\hline & Affordable & 12 & $1.9 \%$ \\
\hline & Job Requirement & 30 & $4.8 \%$ \\
\hline & Family desire & 9 & $1.4 \%$ \\
\hline & Traveling Requirement & 22 & $3.5 \%$ \\
\hline & Protection for the family & 1 & $0.2 \%$ \\
\hline & Preventing covid-19 complication & 1 & $0.2 \%$ \\
\hline & Multiple purposes & 1 & $0.2 \%$ \\
\hline & No purpose & 1 & $0.2 \%$ \\
\hline \multirow{2}{*}{$\begin{array}{l}\text { Presence of side effects post } \\
\text { vaccination }\left(1^{\text {st }} \text { or } 2^{\text {nd }} \text { dose }\right)\end{array}$} & yes & 343 & $54.9 \%$ \\
\hline & No & 282 & $45.1 \%$ \\
\hline \multirow{12}{*}{$\begin{array}{l}\text { Side effects post vaccination } \\
\left(1^{\text {st }} \text { or } 2^{\text {nd }} \text { dose }\right)\end{array}$} & General: & & \\
\hline & Fever & 232 & $67.7 \%$ \\
\hline & Body Tremor & 76 & $22.2 \%$ \\
\hline & Fainting & 3 & $0.9 \%$ \\
\hline & Uterine pain & 1 & $0.3 \%$ \\
\hline & Swelling of mouth and face and neck & 6 & $1.7 \%$ \\
\hline & Systematic: & & \\
\hline & Dermatological $^{1}$ & 231 & $67.3 \%$ \\
\hline & Gastrointestinal $^{2}$ & 129 & $37.6 \%$ \\
\hline & Neurological $^{3}$ & 249 & $72.6 \%$ \\
\hline & Respiratory $^{4}$ & 21 & $6.2 \%$ \\
\hline & Musculoskeletal $^{5}$ & 282 & $82.3 \%$ \\
\hline
\end{tabular}

\footnotetext{
${ }^{1}$ Dermatological side effects including: Itching in other than vaccine site, Pain and swelling in injection site.

${ }^{2}$ Gastrointestinal side effects including: Nausea, Vomiting, Diarrhea, and Abdominal pain.

${ }^{3}$ Neurological side effects including: Headache, Seizure, Sleep disturbance, Numbness, and Visual disturbance.

${ }^{4}$ Respiratory side effects including: Breathing difficulties, Hoarseness, and Throat pain.

${ }^{5}$ Musculoskeletal side effects including: Muscle pain, Chest heaviness, Chest pain, and Back pain.
} 
The Acceptance, Concerns, and Obstacles related to COVID-19 Vaccination among the General Society in Saudi Arabia.

Table 3: Acceptance, concerns, and obstacles related to the vaccination.

\begin{tabular}{llll}
\hline Acceptance, concerns, and obstacles related to the vaccination & No & \% \\
\hline \multirow{2}{*}{ Take the vaccine after the opportunity to take it } & Yes & 371 & $47.9 \%$ \\
& Maybe & 142 & $18.3 \%$ \\
& Yes & 262 & $33.8 \%$ \\
\hline \multirow{2}{*}{ barriers to taking the vaccine } & No & 176 & $22.7 \%$ \\
& Yes & 599 & $77.3 \%$ \\
\hline concerns of the vaccine & No & 538 & $38.4 \%$ \\
& & 862 & $61.6 \%$
\end{tabular}

Table 4: The participants' obstacles that prevent getting the vaccine $(n=324)$.

\begin{tabular}{lcc}
\hline The reported obstacles that prevent getting the vaccine & No & \% \\
\hline 1- Parental refuse & 12 & $3.7 \%$ \\
2- multiple available types of vaccination & 46 & $14.2 \%$ \\
3- There is conflicting information concerning the vaccine's safety & 62 & $19.1 \%$ \\
4- not have a health reason to be vaccinated & 15 & $4.6 \%$ \\
5- lack of information and education about COVID-19 and its & 70 & $21.6 \%$ \\
vaccination and about how to use the application & & \\
6- Taking a long time to obtain an appointment and being far away & 29 & $9.0 \%$ \\
from the centers & & \\
7- Having health problems or allergy & 58 & $17.9 \%$ \\
8- lactating and pregnant women & 17 & $5.2 \%$ \\
9- Covid-19 was previously diagnosed in less than 6 months & 8 & $2.5 \%$ \\
10- others & 7 & $2.2 \%$ \\
\hline
\end{tabular}

Table 5: The participants' concerns regarding

COVID-19 vaccine $(n=1,796)$.

\begin{tabular}{lcc}
\hline The reported concerns of the vaccine & No & \% \\
\hline Misleading ideas and beliefs * & 635 & $35.4 \%$ \\
Health concerns** & 779 & $43.4 \%$ \\
Virus strains*** & 63 & $3.5 \%$ \\
Vaccine trial and efficacy & 213 & $11.8 \%$ \\
Information circulated on social media & 106 & $5.9 \%$
\end{tabular}

* Misleading ideas and beliefs: Ineffective, harmful, targeted for the elimination of humanity, for experiment, for financial gain, distrust, and lack of conviction in vaccination, lack of need, the strangeness of the disease and the strangeness of its spread

**Health concerns: Side effects, it leads to serious diseases, it causes autism, affects genetics, becomes an annual vaccine, leads to death, anaphylaxis, a history of allergies and shortness of breath ***Virus strains: The presence of a new strain, no specific cause of the virus has been known, virus infection after the vaccine 
The Acceptance, Concerns, and Obstacles related to COVID-19 Vaccination among the General Society in Saudi Arabia.

Table 6: Receiving COVID-19 vaccination in association to the demographics data of the participants $(n=1400)$.

\begin{tabular}{|c|c|c|c|c|c|c|}
\hline \multirow{3}{*}{\multicolumn{2}{|c|}{ Socio-demographic data }} & \multicolumn{4}{|c|}{ Received COVID-19 Vaccine } & \multirow{3}{*}{ P-value } \\
\hline & & \multicolumn{2}{|c|}{ Yes } & \multicolumn{2}{|c|}{ No } & \\
\hline & & Freq & $\%$ & Freq & $\%$ & \\
\hline \multirow{2}{*}{ Gender } & Male & 263 & $42.1 \%$ & 253 & $32.6 \%$ & \multirow{2}{*}{$0.002 *$} \\
\hline & Female & 362 & $57.9 \%$ & 522 & $67.4 \%$ & \\
\hline \multirow{3}{*}{ Age in years } & $16-36$ & 259 & $41.4 \%$ & 421 & $54.3 \%$ & \multirow{3}{*}{2.899} \\
\hline & $37-56$ & 252 & $40.3 \%$ & 283 & $36.5 \%$ & \\
\hline & 57 and older & 114 & $18.2 \%$ & 71 & $9.2 \%$ & \\
\hline \multirow{2}{*}{ Nationality } & Saudi & 603 & $96.5 \%$ & 711 & $91.7 \%$ & \multirow{2}{*}{$0.002 *$} \\
\hline & Non-Saudi & 22 & $3.5 \%$ & 64 & $8.3 \%$ & \\
\hline \multirow{4}{*}{$\begin{array}{l}\text { Place of } \\
\text { residence }\end{array}$} & Western Region & 532 & $85.1 \%$ & 605 & $78.1 \%$ & \multirow{4}{*}{$0.001 *$} \\
\hline & $\begin{array}{l}\text { Central Region } \\
\text { Eastern Region }\end{array}$ & $\begin{array}{l}72 \\
11\end{array}$ & $\begin{array}{l}11.5 \% \\
1.8 \%\end{array}$ & $\begin{array}{l}87 \\
34\end{array}$ & $\begin{array}{l}11.2 \% \\
4.4 \%\end{array}$ & \\
\hline & Southern Region & 6 & $1.0 \%$ & 29 & $3.7 \%$ & \\
\hline & Northern Region & 4 & $0.6 \%$ & 20 & $2.6 \%$ & \\
\hline \multirow{4}{*}{$\begin{array}{l}\text { Educational } \\
\text { level }\end{array}$} & $\begin{array}{l}\text { Elementary/ Intermediate } \\
\text { School }\end{array}$ & 18 & $2.9 \%$ & 32 & $4.1 \%$ & \multirow{4}{*}{0.332} \\
\hline & High school/ Diploma & 162 & $25.9 \%$ & 215 & $27.7 \%$ & \\
\hline & Bachelor's degree & 351 & $56.2 \%$ & 430 & $55.5 \%$ & \\
\hline & Master or higher degree & 94 & $15.0 \%$ & 98 & $12.6 \%$ & \\
\hline \multirow{3}{*}{$\begin{array}{l}\text { Work in } \\
\text { government } \\
\text { sector }\end{array}$} & Yes & 217 & $34.7 \%$ & 212 & $27.4 \%$ & \multirow{3}{*}{$0.011 *$} \\
\hline & No & 171 & $27.4 \%$ & 229 & $29.5 \%$ & \\
\hline & Do not work & 237 & $37.9 \%$ & 334 & $43.1 \%$ & \\
\hline \multirow{2}{*}{$\begin{array}{l}\text { History of } \\
\text { COVID-19 }\end{array}$} & Yes & 56 & $9.0 \%$ & 148 & $19.1 \%$ & \multirow[t]{2}{*}{9.087} \\
\hline & No & 569 & $91.0 \%$ & 627 & $80.9 \%$ & \\
\hline
\end{tabular}

Note: P: Pearson $X^{2}$ test $\quad * P<0.05$

(significant).

Table 7: Participants' acceptance to take the vaccine $(\mathrm{n}=1400)$.

Acceptance Percentage \%

Vaccinated or willing $\quad 996(71.1 \%)$

to vaccinate

\begin{tabular}{cc}
\hline Hesitancy & $262(18.7 \%)$ \\
\hline Refusing vaccination & $142(10.2 \%)$
\end{tabular}


The Acceptance, Concerns, and Obstacles related to COVID-19 Vaccination among the General Society in Saudi Arabia.

Table 8: Different types of barriers were involved in the study.

\section{Barriers}

Vaccine variety types and Covid19 strains issues

Safety issues

Health issues

Convincement of beneficial issues

Vaccine information issue

Vaccination application issue

Family Issues

Distance and transport to centres issue

Covid 19 infected

Pregnancy

Breastfeeding

Waiting time issues

Side effects issues

Allergy

Vaccine production issues

Medication Issues

Religious Believes
Percentage \%

$62(35.2 \%)$

$59(33.5 \%)$

$56(32 \%)$

$46(26.1 \%)$

$25(14.2)$

$19(10.8 \%)$

$14(7.9 \%)$

$9(5.2 \%)$

$8(4.8 \%)$

$7(4.2 \%)$

$7(4.8 \%)$

$6(3.4 \%)$

$4(2.4 \%)$

$3(1.8 \%)$

$1(0.6 \%)$

$1(0.6)$

$1(0.6 \%)$ 
This comparison shows that Arab countries have more concerns about the COVID-19 vaccine than the majority of the rest of the world. Although our study represents one of the first assessments of COVID-19 vaccination behavior in KSA, we acknowledge several limitations. One of these is related to convenient sample conducted on an online platform which may limits the generalizability of the study's results. Additionally, reporting bias could be possible since the study depends on self-reported information that might be influenced by participants' opinions and standards in understanding the items or their proclivity to express their feelings in a certain manner. Lastly, the current study used a cross-sectional design which precludes the ability to make causal conclusions.

\section{Conclusion}

In conclusion, the current study revealed that the residents of Saudi Arabia had low level of compliance in regard to receive different types of vaccination including flu vaccine and meningitis vaccine. In addition, Female participants showed high levels of compliance to receive COVID-19 vaccination. Knowledge and awareness about COVID-19 are still lacking. Thus, a targeted health educational programs are needed to increase the individual convince and reduce the fears about vaccination, which will contribute to increase the rates of receiving COVID19 vaccine in the future.

\section{List of Abbreviations:}

COVID-19: Coronavirus Disease 2019, SARS-CoV2: Severe Acute Respiratory Syndrome Coronavirus 2, WHO: World Health Organization, NRVV: nonreplicating viral vectors, mRNA: messenger ribonucleic acid, US: United States, KSA: Kingdome of Saudi Arabia, CI: Confidence Interval, IRB: Institutional Review Board, UQU: Umm Al-Qura University, SPSS: Statistical Package for the Social Sciences.

\section{Conflict of Interest}

None

\section{Funding}

None

\section{References:}

1. Liu Y-C, Kuo R-L, Shih S-R. COVID-19: The first documented coronavirus pandemic in history. Biomed J. 2020;43(4):328-33.

2. Baloch S, Baloch MA, Zheng T, Pei X. The coronavirus disease 2019 (COVID-19) pandemic. Tohoku J Exp Med. 2020;250(4):271-8.

3. Tang B, Bragazzi NL, Li Q, Tang S, Xiao Y, Wu J. An updated estimation of the risk of transmission of the novel coronavirus (2019-nCov). Infect Dis Model. 2020;5:248-55.

4. Chen N, Zhou M, Dong X, Qu J, Gong F, Han Y, et al. Epidemiological and clinical characteristics of 99 cases of 2019 novel coronavirus pneumonia in Wuhan, China: a descriptive study. Lancet (London, England). 2020 Feb;395(10223):507-13.

5. Fisk RJ. Barriers to vaccination for coronavirus disease 2019 (COVID-19) control: experience from the United States. Glob Heal J [Internet]. 2021; Available from: https://www.sciencedirect.com/science/article/pii/S24 14644721000051

6. Liu X, Liu C, Liu G, Luo W, Xia N. COVID-19: Progress in diagnostics, therapy and vaccination. Theranostics. 2020;10(17):7821.

7. Sharma O, Sultan AA, Ding H, Triggle CR. A Review of the Progress and Challenges of Developing a Vaccine for COVID-19. Front Immunol. 2020;11:585354.

8. Izda V, Jeffries MA, Sawalha AH. COVID-19: A review of therapeutic strategies and vaccine candidates. Clin Immunol [Internet]. 2021;222:108634. Available from: https://www.sciencedirect.com/science/article/pii/S15 21661620307944

9. Lazarus J V, Ratzan SC, Palayew A, Gostin LO, Larson HJ, Rabin K, et al. A global survey of potential acceptance of a COVID-19 vaccine. Nat Med [Internet]. 2021;27(2):225-8. Available from: https://doi.org/10.1038/s41591-020-1124-9

10. Dean AG, Sullivan KM SM. OpenEpi: Open Source Epidemiologic Statistics for Public Health, Version 3.01 [Internet]. [cited 2021 Jun 25]. Available from: www.OpenEpi.com

11. General Authority for Statistics. Reports and Statistics [Internet]. Unified National Platform. 2021. Available from: https://www.my.gov.sa/wps/portal/snp/aboutksa/saud iReportsAndStatistics

12. Menni C, Klaser K, May A, Polidori L, Capdevila J, Louca P, et al. Vaccine side-effects and SARS-CoV2 infection after vaccination in users of the COVID Symptom Study app in the UK: a prospective observational study. Lancet Infect Dis [Internet]. 2021;21(7):939-49. Available from: https://www.sciencedirect.com/science/article/pii/S14 73309921002243

13. Alhazmi A, Alamer E, Daws D, Hakami M, Darraj M, Abdelwahab S, et al. Evaluation of Side Effects Associated with COVID-19 Vaccines in Saudi Arabia. Vaccines. 2021;9(6):674. 
The Acceptance, Concerns, and Obstacles related to COVID-19 Vaccination among the General Society in Saudi Arabia.

14. Malik AA, McFadden SM, Elharake J, Omer SB. Determinants of COVID-19 vaccine acceptance in the US. EClinicalMedicine. 2020 Sep;26:100495.

15. Al-Mohaithef M, Padhi BK. Determinants of COVID-19 Vaccine Acceptance in Saudi Arabia: A Web-Based National Survey. J Multidiscip Healthc. 2020;13:1657-63.

16. Saied SM, Saied EM, Kabbash IA, Abdo SAE-F. Vaccine hesitancy: Beliefs and barriers associated with COVID-19 vaccination among Egyptian medical students. J Med Virol. 2021 Jul;93(7):4280-91.

17. Pollak Y, Shoham R, Dayan H, Seri OG, Berger I. Background and concurrent factors predicting nonadherence to public health preventive measures during the chronic phase of the COVID-19 pandemic. medRxiv. 2020;2(4):32.

18. Arber BSc Sara MsP, Ginn BSc Jay MsP. Gender differences in informal caring. Health Soc Care Community [Internet]. 1995 Jan 1;3(1):19-31. Available from: https://doi.org/10.1111/j.13652524.1995.tb00003.x

19. Alshammary F, Siddiqui AA, Amin J, Ilyas M, Rathore HA, Hassan I, et al. Prevention knowledge and its practice towards COVID-19 among general population of Saudi Arabia: a gender-based perspective. Curr Pharm Des. 2021;27(13):1642-8.

20. Kaadan MI, Abdulkarim J, Chaar M, Zayegh O, Keblawi MA. Determinants of COVID-19 vaccine acceptance in the Arab world: a cross-sectional study. Glob Heal Res policy. 2021 Jul;6(1):23. 